\title{
Perturbative solution of a propagating interface in the phase field model
}

\author{
Mao Hiraizumi ${ }^{1}$, Shin-ichi Sasa ${ }^{2}$ \\ Department of Physics, Kyoto University, Kyoto 606-8502, Japan \\ E-mail: hiraizumi.mao.72s@st.kyoto-u.ac.jp ${ }^{1}$, sasa@scphys.kyoto-u.ac.jp ${ }^{2}$
}

\begin{abstract}
When a stable ordered phase and a metastable disordered phase are separated by a flat interface, the metastable state changes to the stable state through the propagation of the interface. For cases in which latent heat is generated, the interface displacement during some time interval is proportional to the square root of the time interval when the extent of supercooling is less than a certain value. We demonstrate this behavior by deriving a perturbative solution for a propagating interface in the phase field model. We calculate the leading-order contribution explicitly, and find that the interface temperature deviates from the equilibrium transition temperature in proportion to the interface velocity.
\end{abstract}

\section{Introduction}

Systems in which different phases coexist, such as growing crystal, exhibit a rich variety of dynamical behaviors [1, 2]. The simplest situation is that a flat interface connects a stable ordered phase and a metastable disordered phase. In this case, the metastable state becomes stable through the propagation of the interface. The phenomenon can be described by the time evolution of an order-parameter field that represents the extent of the order.

When the order parameter is the only relevant dynamical variable of the system, the solution of the propagating interface is easily determined [3]. However, as typically observed in crystallization, energy flow becomes a significant physical quantity, because latent heat is generated in the ordering process. In this case, a temperature field also evolves under the influence of the generated latent heat, and this temperature field influences the time evolution of the order parameter. The set of coupled equations is called the phase field model [4, 5, 6, 7, 8, 9]. Deriving a solution for the propagating interface in the phase field model is not a simple task.

Explicitly, let $T_{s}$ and $T_{m}$ be the temperatures of the heat baths attached to the stable ordered phase in the left region and to the metastable phase in the right region, respectively. Note that $T_{s}$ and $T_{m}$ are less than the equilibrium transition temperature $T_{c}$. The latent heat per unit volume is denoted by $L$ and the specific heat at constant 
pressure per unit volume is denoted by $c_{p}$. An important dimensionless quantity is

$$
\Delta \equiv \frac{c_{p}\left(T_{c}-T_{m}\right)}{L}
$$

which represents the extent of the supercooling. The interface position $R(t)$ at time $t$ depends on $\Delta$. Specifically, numerical simulations of the phase field model show that $R(t) \simeq t^{1 / 2}$ for the case $\Delta<1$, while $R(t) \simeq t$ for the case $\Delta>1$ [10].

A theoretical problem is to derive this numerical observation by analyzing the phase field model. For the case $\Delta>1$, there is a solution describing $R(t) \simeq t[11$. We note that $R(t) \simeq t^{1 / 2}$ was derived for the case $\Delta<1$ in the Stefan model, which formulates a dynamic boundary value problem [12]. However, to the best of our knowledge, there have been no theoretical studies of the case $\Delta<1$ in the phase field model.

In this paper, we derive a perturbative solution of the propagating interface for the case $\Delta<1$ in the phase field model. A key step in deriving the solution is that the solution is assumed to have a scaling form, with two scaled coordinates and one dimensionless time-dependent small parameter. Expanding the solution in the small parameter, we determine the leading-order and next-order contributions to the solution. In particular, we obtain

$$
R(t)=\sqrt{2 D C t},
$$

where $R(0)=0, D$ is a thermal diffusion constant, and $C$ is a constant determined by the boundary conditions of the temperature field. As a remarkable property, $C$ exhibits divergent behavior as $\Delta \rightarrow 1$ from below. The solution also shows that the interface temperature deviates from the equilibrium transition temperature in proportion to the interface velocity.

\section{Phase field model}

Since we are focusing on the motion of a flat interface in a three-dimensional space, we study a one-dimensional system. Let $\phi(x, t)$ be an order-parameter field that represents the ordered (e.g., solid) phase by $\phi(x, t)=1$ and the disordered (e.g., liquid) phase by $\phi=0$. The phase field model is a set of coupled equations for the order-parameter field $\phi(x, t)$ and the temperature field $T(x, t)$. We first assume a free energy density $f(\phi, T)$ for a given material, where $f(\phi, T)$ takes a double-well form that possesses local minima at $\phi=0$ and $\phi=1$ for each $T$. The transition temperature $T_{c}$ is determined such that $f(\phi, T)$ is minimized at $\phi=1$ when $T<T_{c}$, while $f(\phi, T)$ is minimized at $\phi=0$ when $T>T_{c}$. We then define the free energy functional $F[\phi, T]$ as

$$
F[\phi, T]=\int d x\left(f(\phi, T)+\frac{\xi^{2}}{2}\left(\partial_{x} \phi\right)^{2}\right),
$$

where $\xi$ is a parameter that represents the interface width. The gradient term becomes

relevant in the interface region, corresponding to the surface free energy. We assume that $\phi$ evolves so that the free energy decreases. That is, the equation of $\phi$ is given as

$$
\tau \partial_{t} \phi=-\frac{\delta F[\phi, T]}{\delta \phi(x)}
$$


where $\tau$ characterizes the time scale of $\phi$.

From the law of enthalpy conservation at constant pressure, the equation for $T$ is determined as

$$
\partial_{t} T=D \partial_{x}^{2} T+\frac{L}{c_{p}} \frac{\partial \phi}{\partial t}
$$

where the thermal diffusion constant $D$ is assumed to be independent of $(T, \phi)$. The first term on the right-hand side of (5) represents heat diffusion and the second term represents the effect of the latent heat generated by the time evolution of $\phi$. To simplify the notation, we introduce a dimensionless temperature

$$
\theta(x, t) \equiv \frac{c_{p}\left(T(x, t)-T_{c}\right)}{L}
$$

and define

$$
\bar{f}(\phi, \theta) \equiv f\left(\phi, T_{c}+\frac{L}{c_{p}} \theta\right) .
$$

Using these quantities, we can rewrite (41) and (5) as

$$
\begin{aligned}
\tau \partial_{t} \phi & =\xi^{2} \partial_{x}^{2} \phi-\frac{\partial \bar{f}}{\partial \phi} \\
\partial_{t} \theta & =D \partial_{x}^{2} \theta+\partial_{t} \phi .
\end{aligned}
$$

We study cases in which a stable ordered phase (e.g., crystal) in the left-region grows in a metastable disordered phase (e.g., supercooled liquid) in the right region. Thus, we impose the following boundary conditions on $\phi$ and $\theta$ :

$$
\begin{aligned}
& \lim _{x \rightarrow-\infty} \phi(x, t)=1, \\
& \lim _{x \rightarrow \infty} \phi(x, t)=0,
\end{aligned}
$$

and

$$
\begin{aligned}
& \lim _{x \rightarrow-\infty} \theta(x, t)=-\Pi, \\
& \lim _{x \rightarrow \infty} \theta(x, t)=-\Delta,
\end{aligned}
$$

where $\Delta$ is defined as (11) and $\Pi$ is

$$
\Pi \equiv-\frac{c_{p}\left(T_{s}-T_{c}\right)}{L}
$$

Note that $\Pi \geq 0$. More precisely, we assume that $\phi(x, t)$ and $\theta(x, t)$ converge faster than an exponential form as a function of $x$ in the limit $|x| \rightarrow \infty$.

\section{Results}

We construct a solution of (8) and (9) with the boundary conditions (10), (11), (12), and (13). Let $R(t)$ be the interface position at time $t$, which satisfies $\phi(R(t), t)=0.5$. First, we note that $\xi$ and $D / \dot{R}$ provide length scales of the solution $\phi(x, t)$ and $\theta(x, t)$, 
respectively, where $\dot{R}(t)=d R(t) / d t$. Based on this observation, we define the scaled coordinates

$$
\begin{aligned}
& w \equiv \frac{x-R(t)}{\xi}, \\
& z \equiv \frac{\dot{R}(x-R(t))}{D} .
\end{aligned}
$$

We also introduce a small dimensionless time-dependent quantity

$$
\eta \equiv \frac{\xi \dot{R}}{D}
$$

Using $w, z$, and $\eta$, we assume that the solution takes the scaling form

$$
\begin{aligned}
& \phi(x, t)=\Phi(w ; \eta), \\
& \theta(x, t)=\Theta(z ; \eta) .
\end{aligned}
$$

Focusing on cases where $\eta(t) \ll 1$, we expand the solution in $\eta$ as

$$
\begin{aligned}
& \Phi(w ; \eta)=\Phi_{0}(w)+\eta \Phi_{1}(w)+O\left(\eta^{2}\right), \\
& \Theta(z ; \eta)=\Theta_{0}(z)+\eta \Theta_{1}(z)+O\left(\eta^{2}\right) .
\end{aligned}
$$

Substituting (20) and (21) into (8) and extracting terms that are independent of $\eta$, we have

$$
\partial_{w}^{2} \Phi_{0}-\frac{\partial \bar{f}}{\partial \phi}\left(\Phi_{0}, \Theta_{0}(0)\right)=0
$$

where we have used $z=\eta w$. Multiplying both sides of (22) by $\partial_{w} \Phi_{0}$ and integrating the result from $-\infty$ to $+\infty$, we obtain

$$
\bar{f}\left(1, \Theta_{0}(0)\right)=\bar{f}\left(0, \Theta_{0}(0)\right),
$$

which leads to

$$
\Theta_{0}(0)=0 \text {. }
$$

By solving (22) with the boundary conditions (10) and (11), we can determine the unique solution $\Phi_{0}(z)$.

Next, substituting (20) and (21) into (9) and extracting the leading-order terms in $\eta$, we obtain

$$
\left(\frac{\ddot{R}}{\dot{R}} z-\frac{\dot{R}^{2}}{D}\right) \partial_{z} \Theta_{0}=\frac{\dot{R}^{2}}{D} \partial_{z}^{2} \Theta_{0}+\frac{\dot{R}^{2}}{D} \delta(z),
$$

where $\ddot{R}=d^{2} R(t) / d t^{2}$; here, we have used

$$
\left.\lim _{\eta \rightarrow 0} \frac{1}{\eta} \partial_{w} \Phi_{0}(w)\right|_{w=\frac{z}{\eta}}=-\delta(z) .
$$

By the method of separation of variables, we find

$$
\frac{D \ddot{R}}{\dot{R}^{3}}=-\frac{1}{C}
$$


with a constant $C$. From this relation, we obtain (2). Then, (25) becomes

$$
\partial_{z}^{2} \Theta_{0}+\frac{1}{C}(z+C) \partial_{z} \Theta_{0}=-\delta(z)
$$

We now analyze (28). First, set $\Theta^{+}(z)=\Theta(z)$ for $z>0$ and $\Theta^{-}(z)=\Theta(z)$ for $z<0$. Solving (28), we obtain

$$
\begin{aligned}
\Theta_{0}^{ \pm}(z) & =\left.\partial_{z} \Theta_{0}\right|_{z= \pm 0} e^{\frac{C}{2}} \int_{0}^{z} e^{-\frac{\left(z^{\prime}+C\right)^{2}}{2 C}} d z^{\prime} \\
& =\left.\partial_{z} \Theta_{0}\right|_{z= \pm 0} \sqrt{\frac{\pi C}{2}} e^{\frac{C}{2}}\left(\operatorname{erf}\left(\frac{\mathrm{z}}{\sqrt{2 \mathrm{C}}}+\sqrt{\frac{\mathrm{C}}{2}}\right)-\operatorname{erf}\left(\sqrt{\frac{\mathrm{C}}{2}}\right)\right)
\end{aligned}
$$

with the connection condition

$$
\left.\partial_{z} \Theta_{0}^{+}\right|_{z=+0}-\left.\partial_{z} \Theta_{0}^{-}\right|_{z=-0}=-1 .
$$

For later convenience, we set

$$
\left.A \equiv \partial_{z} \Theta^{-}\right|_{z=-0}
$$

Using the boundary conditions (12) and (13), we derive

$$
\begin{aligned}
& \Delta=(1-A) \sqrt{\frac{\pi C}{2}} e^{\frac{C}{2}}\left(1-\operatorname{erf}\left(\sqrt{\frac{\mathrm{C}}{2}}\right)\right), \\
& \Pi=A \sqrt{\frac{\pi C}{2}} e^{\frac{C}{2}}\left(1+\operatorname{erf}\left(\sqrt{\frac{\mathrm{C}}{2}}\right)\right) .
\end{aligned}
$$

Therefore, for a given value of $(\Delta, \Pi)$ satisfying $\Delta<1$, we can determine $A$ and $C$. As one example, we consider the case $\Pi=0$. In this case, we have $A=0$ from (34), and we obtain $C$ as a function of $\Delta$. The result is displayed in Fig. 1. Note that $C \rightarrow \infty$ as $\Delta \rightarrow 1$.
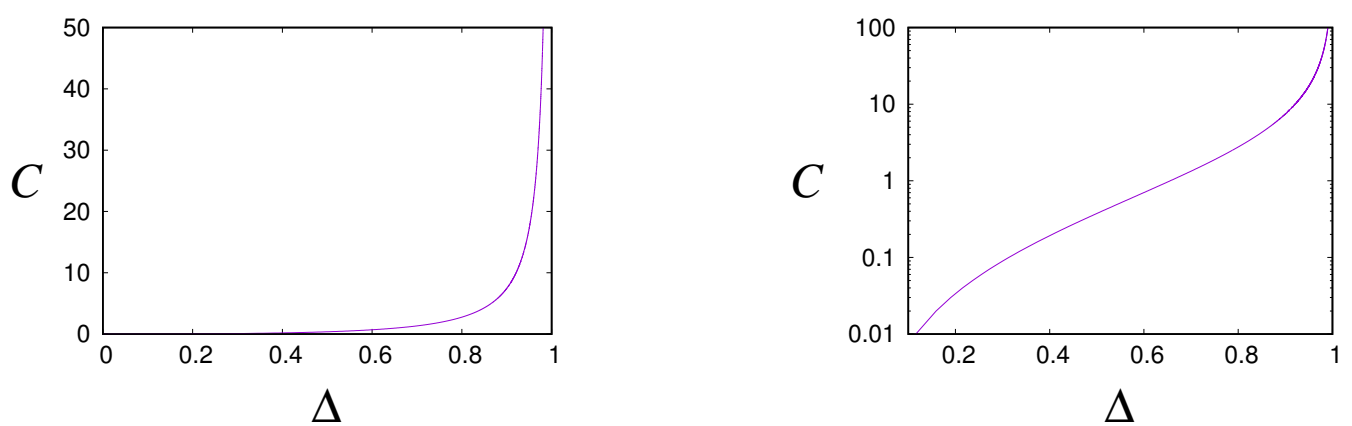

Fig $1 . C$ as a function of $\Delta$ for the case $\Pi=0$. Normal and log plots are displayed on the left and right sides, respectively. $C \rightarrow \infty$ as $\Delta \rightarrow 1$.

Furthermore, we substitute (20) and (21) into (8) and (9) and collect terms proportional to $\eta$, where we also assume the expansion

$$
\frac{D \ddot{R}}{\dot{R}^{3}}=-\frac{1}{C}-C_{1} \eta+O\left(\eta^{2}\right) \text {. }
$$

We then obtain equations for $\Phi_{1}(w)$ and $\Theta_{1}(z)$. By solving these equations with the boundary conditions that $\Phi_{1}(w)$ and $\Theta_{1}(z)$ converge to zero faster than an exponential 
form in the limit $|w| \rightarrow \infty$ and $|z| \rightarrow \infty$, we can determine $\Phi_{1}(w), \Theta_{1}(z)$, and $C_{1}$. (See Appendix A.) For example, the interface temperature $T(R(t), t)$ is determined as

$$
T(R(t), t)=T_{c}+\frac{L \xi \dot{R}(t)}{c_{p} D} \Theta_{1}(0)
$$

with the formula for $\Theta_{1}(0)$ given in $(\underline{A .3})$. This means that the interface temperature deviates from the equilibrium transition temperature and the deviation is proportional to the interface velocity $\dot{R}$.

\section{Concluding remarks}

We have studied the deterministic equation describing phase coexistence. However, because the interface length $\xi$ is less than a micrometer, stochastic processes may become relevant in the interface region. To consider such effects, we examine an energyconserving Potts model with kinetic energy as a natural extension of energy-conserving kinetic Ising models [13, 14, 15, 16. We will report the behavior in a separate paper.

In this paper, we focused on the propagation of a flat interface as the simplest case. In some experiments, flat interfaces have exhibited the Mullins-Sekerka instability [17], which leads to a rich variety of patterns [1]. The instability of propagating interfaces was studied in the phase field model [18, 19], including the instability of solutions with $R(t) \simeq t^{1 / 2}$ [20]. With regard to the propagation velocity of a destabilized interface, it was reported that $R(t) \simeq t$ when $\Delta>1$ in the one-sided model [21], while a theoretical analysis of the Stefan-type model shows $R(t) \simeq t^{1 / 2}$ even when $\Delta>1$ [22]. A systematic understanding of these phenomena will be studied in the future.

\section{Acknowledgments}

This work was supported by KAKENHI (Grant Nos. 17H01148, 19H05795, and 20K20425).

\section{Appendix A. Next-order contribution}

In this appendix, we calculate the next-order contribution to the solution. To simplify the notation, we introduce a dimensionless parameter

$$
\alpha \equiv \frac{D \tau}{\xi^{2}},
$$

which represents the ratio of the thermal diffusion constant to the order-parameter diffusion constant.

First, we substitute (20) and (21) into (81). We collect terms proportional to $\eta$, noting that $\dot{\eta}=O\left(\eta^{3}\right)$ from (27). We then obtain

$$
-\alpha \partial_{w} \Phi_{0}(w)=\partial_{w}^{2} \Phi_{1}-\Phi_{1} \frac{\partial^{2} \bar{f}}{\partial \phi^{2}}\left(\Phi_{0}, 0\right)
$$




$$
-\frac{\partial^{2} \bar{f}}{\partial \phi \partial \theta}\left(\Phi_{0}, 0\right)\left(w \Theta_{0}^{\prime}( \pm 0)+\Theta_{1}(0)\right)
$$

where either +0 or -0 is selected in the second line depending on whether $w>0$ or $w<0$. Multiplying both sides of $(\underline{\mathrm{A} .2})$ by $\partial_{w} \Phi_{0}$ and integrating the result from $-\infty$ to $\infty$, we obtain

$$
-\alpha I=-\Theta_{1}(0) J+(1-A) K_{+}-A K_{-}
$$

with

$$
\begin{aligned}
I & \equiv \int_{-\infty}^{\infty} d w\left(\partial_{w} \Phi(w)\right)^{2}, \\
J & \equiv \int_{-\infty}^{\infty} d w \frac{\partial^{2} \bar{f}}{\partial \phi \partial \theta}(\Phi, 0) \partial_{w} \Phi, \\
K_{ \pm} & \equiv \pm \int_{0}^{ \pm \infty} d w w \frac{\partial^{2} \bar{f}}{\partial \phi \partial \theta}(\Phi, 0) \partial_{w} \Phi .
\end{aligned}
$$

Here, we have also used (32). Equation (A.3) determines the value of $\Theta_{1}(0)$, and corresponds to the solvability condition for the equation of $\Phi_{1}(w)$. Then, $\Phi_{1}(w)$ can be obtained as the solution of the linear equation (A.2).

Next, we substitute (20) and (21) into (9). Collecting terms proportional to $\eta$, we obtain

$$
\partial_{z}^{2} \Theta_{1}+\partial_{z} \Theta_{1}+\frac{1}{C} \partial_{z}\left(z \Theta_{1}\right)=C_{1} z \partial_{z} \Theta_{0}
$$

By integrating (A.7) from $-\infty$ to $+\infty$, we have

$$
C_{1}=0
$$

which corresponds to the solvability condition for the equation of $\Theta_{1}(z)$. Then, (A.7) becomes

$$
\partial_{z} \Theta_{1}+\frac{1}{C}(z+C) \Theta_{1}=B
$$

where $B$ is a constant. Because $\Theta_{1}(z)$ converges to zero faster than an exponential form as a function of $|z|$ in the limit $|z| \rightarrow \infty$, we find that

$$
B=0 \text {. }
$$

We then solve $(\mathrm{A} .9$ ) as

$$
\Theta_{1}(z)=\Theta_{1}(0) e^{\frac{C}{2}} e^{-\frac{(z+C)^{2}}{2 C}}
$$

where $\Theta_{1}(0)$ is given by $(A .3)$.

\section{References}

[1] Langer J S 1980 Instabilities and pattern forestation in crystal growth Rev. Mod. Phys. 521

[2] Ben-Jacob E and Garik P 1990 The formation of patterns in nonequilibrium growth Nature 343 523

[3] Pomeau Y 1986 Front motion, metastability and subcritical bifurcations in hydrodynamics Physica D 233 
[4] Fix G J 1983 Phase field methods for free boundary problems Free Boundary Problems: Theory and Applications ed Fasano A and Primicerio M vol 2 (Piman, Boston) p 580

[5] Caginalp G 1984 Surface tension and supercooling in solidification theory Springer Lecture Notes in Physics, Applications of Field Theory to Statistical Mechanics (Springer, Berlin) p 216

[6] Langer J S 1986 Models of pattern formation in first-order phase transitions Directions in Condensed Matter Physics ed Grinstein G and Mazenko G (World Scientific, Philadelphia) p 165

[7] Collins J B and Levine H 1986 Diffuse interface model of diffusion-limited crystal growth Phys. Rev. B 332020

[8] Penrose O and Fife P C 1990 Thermodynamically consistent models of phasefield type for the kinetics of phase transitions Physica D 4344

[9] Kobayashi R 1993 Modeling and numerical simulations of dendritic crystal growth Physica D 63 410

[10] Löwen H, Bechhoefer J and Tuckerman L S 1992 Crystal growth at long times: critical behavior at the crossover from diffusion to kinetics-limited regimes Phys. Rev. A 452399

[11] Caginalp G and Nishiura Y 1991 The existence of traveling waves for phase field equations and convergence to sharp interface models in the singular limit Quart. Appl. Math. 49147

[12] Dewynne J N, Howison S D, Ockendon J R and Xie W 1989 Asymptotic behavior of solutions to the Stefan problem with a kinetic condition at the free boundary J. Austral. Math. Soc. Ser. B 3181

[13] Kadanoff L and Swift J 1968 Transport coefficients near the liquid-gas critical point Phys. Rev. 165310

[14] Creutz M 1983 Microcanonical monte carlo simulation Phys. Rev. Lett. 501411

[15] Casartelli M, Macellari N and Vezzani A 2007 Heat conduction in a two-dimensional Ising model Eur. Phys. J. B 56, 149

[16] Hiraizumi M, Ohta H and Sasa S in preparation.

[17] Mullins W W and Sekerka R F 1964 Stability of a planar interface during solidification of a dilute binary alloy J. Appl. Phys. 35D 444

[18] Kupfermann R, Shochet O, Ben-Jacob E and Schuss Z 1992 Phase-field model: boundary layer, velocity of propagation, and the stability spectrum Phys. Rev. B 4616045

[19] Braun R J, McFadden G B and Coriell S R 1994 Morphological instability in phase-field models of soliddication Phys. Rev. E $\mathbf{4 9} 4336$

[20] Lambert R A and Rangel R H 2002 Linear stability analysis of the solidification of a supercooled liquid in a half-space Int. J. Heat Mass Transf. 454577

[21] Jasnow D and Vials J 1990 Dynamical scaling during interfacial growth in the one-sided model Phys. Rev. A 416910

[22] Caginalp G 1999 Dynamical renormalization group calculation of a two-phase sharp interface model Phys. Rev. E 60 R6267 